Pacific Journal of Mathematic 


\section{ENTROPY OF SELF-HOMEOMORPHISMS OF STATISTICAL PSEUDO-METRIC SPACES}

\section{AlaN SALESKI}

A pseudo-Menger space is a set $X$ together with a function $\theta: X \times X \rightarrow \mathscr{D}$, the set of distribution functions, satisfying certain natural axioms similar to those of a pseudo-metric space. Let $T: X \rightarrow X$ be a bijection and let $\theta_{T}$ denote the topology generated by $\left\{T^{i} U(p, \varepsilon, \lambda): i \in Z, p \in X, \varepsilon>0, \lambda>0\right\}$ where $U(p, \varepsilon, \lambda)=\{q: \theta(p, q)(\varepsilon)>1-\lambda\}$. Assume that $\theta_{T}$ is compact. Let $h(T, \theta)$ denote the topological entropy of $T$ with respect to the $\theta_{T}$ topology. The purpose of this note is to show that if one is given a sequence $\left\{\theta_{n}\right\}$ of pseudo-Menger structures on $X$ satisfying $\theta_{n}(p, q) \geqq \theta(p, q)$ and $\theta_{n}(p, q) \rightarrow \theta(p, q)$ in distribution for all $p, q \in X$ then $h\left(T, \theta_{n}\right) \rightarrow h(T, \theta)$. A counterexample is then given to show that, in general, the condition $\theta_{n}(p, q) \geqq \theta(p, q)$ cannot be removed.

1. The investigation of statistical metric spaces was undertaken by Karl Menger [5] in 1942. Essentially these are spaces in which the "distance" between any two points is given by a probability distribution function. Our purpose is to investigate the behavior of the topological entropy of a self-homeomorphism of a compact Menger space under perturbations of these distribution functions. We proceed to give precise definitions.

2. Preliminaries. Let $I$ denote the closed unit interval, $Q^{+}$the positive rationals, $Z^{+}$the positive integers, and $\mathscr{D}$ the set of all leftcontinuous monotone increasing functions $F: R \rightarrow I$ satisfying $F(0)=0$ and $\sup F(x)=1$. Let $H$ be the function defined by: $H(t)=0$ for $t \leqq 0$ and $H(t)=1$ for $t>0$.

Throughout our discussion, $X$ will be a fixed set. Let $\mathscr{F}$ denote the collection of all functions $\theta: X \times X \rightarrow \mathscr{D}$. For convenience we shall often write $\theta_{p q}$ in place of $\theta(p, q)$. A statistical pseudo-metric space is an ordered pair $(X, \theta)$ where $\theta \in \mathscr{F}$ satisfies

(a) $\theta_{p q}=\theta_{q p}$ for all $p, q \in X$.

(b) $\theta_{p q}(a+b)=1$ whenever $\theta_{p r}(a)=\theta_{r q}(b)=1$.

(c) $\theta_{p p}=H$ for all $p \in X$.

If, in addition, $\theta$ satisfies

(d) $\theta_{p q}=H$ only if $p=q$

then $(X, \theta)$ is a statistical metric space.

Let $\mathscr{S}$ denote the collection of all $\theta$ for which $(X, \theta)$ is a statistical pseudo-metric space.

A triangular norm is a function $\Delta: I \times I \rightarrow I$ which is associative, 
commutative, nondecreasing in each variable and satisfies $\Delta(y, 1)=y$ for each $y \in I$. A continuous Menger space [pseudo-Menger space] is a statistical metric space [statistical pseudo-metric space] $(X, \theta)$ for which there exists a continuous triangular norm $\Delta$ satisfying:

(e) $\theta_{p r}(a+b) \geqq \Delta\left(\theta_{p q}(a), \theta_{q r}(b)\right)$ for all $p, q, r \in X$ and all $a, b \in \boldsymbol{R}$.

Let $\mathscr{C l}$ denote the set of all $\theta$ for which $(X, \theta)$ is a continuous pseudo-Menger space.

If $\theta \in \mathscr{F}$ then one defines a topology on $X, \tau(\theta)$, in the following manner: If $p \in X, \varepsilon, \lambda>0$, let $U(p, \varepsilon, \lambda, \theta)=\left\{q \in X: \theta_{p q}(\varepsilon)>1-\lambda\right\}$. These sets are a subbasis for $\tau(\theta)$. It was proven in [9] that if $(X, \theta)$ is a continuous Menger space then $\tau(\theta)$ is metrizable.

We shall be concerned with studying a bijective map $T: X \rightarrow X$. If $\tau$ is a topology on $X$ then $\tau^{T}$ will denote the topology generated by $\left\{T^{i} A: A \in \tau, i \in Z\right\}$. We will say that a map $\theta \in \mathscr{F}$ is T-admissible if $\tau(\theta)^{T}$ is compact and $\theta\left(T^{i} x, T^{i} y\right)=H(i \in Z)$ whenever $\theta_{x y}=H$.

Let $\mathscr{F}_{0}, \mathscr{S}_{0}, \mathscr{C}_{0}$ denote the $T$-admissible maps belonging to $\mathscr{F}$, $\mathscr{S}, \mathscr{L}$ respectively. Of course $\mathscr{L}_{0} \subset \mathscr{S}_{0} \subset \mathscr{F}_{0}$. We define a partial order on $\mathscr{F}_{0}$ as follows: If $\theta, \Psi \in \mathscr{F}_{0}$ then $\theta \leqq \Psi$ if and only if $\theta_{p q}(t) \leqq \Psi_{p q}(t)$ for all $t \in R$ and $p, q \in X$. If $\left\{\theta^{n}: n \in Z^{+}\right\}$and $\Psi$ belong to $\mathscr{F}$ then $\theta^{n} \stackrel{D}{\rightarrow} \Psi$ will mean that for every $(p, q) \in X \times X, \theta_{p q}^{n}(y) \rightarrow$ $\Psi_{p q}(y)$ for each $y \in X$ at which $\Psi_{p q}$ is continuous.

For definitions and properties of measure theoretic entropy the reader is referred to [2] or [6]. If $\Sigma$ is a $\sigma$-algebra of subsets of $X$, $\mu$ an invariant measure on $\Sigma, T \Sigma=\Sigma(\bmod 0)$ and $\Gamma$ a sub- $\sigma$-algebra of $\Sigma$ then $h_{\mu}(T, \Gamma)$ will denote the measure theoretic entropy of $\left(X, \mathrm{~V}_{-\infty}^{\infty} T^{i} \Gamma, \mu, T\right)$.

For definitions and properties of topological entropy we refer the reader to [1] and [3]. If $\tau$ is a compact topology on $X$ for which $T^{-1}(\tau) \subset \tau$ then $h(T, \tau)$ will denote the topological entropy of $(X, \tau, T)$. In case $\theta \in \mathscr{C}_{0}$ we shall write $h(T, \theta)$ in place of $h\left(T, \tau(\theta)^{T}\right)$.

If $\tau$ is a topology on $X$ then $\sigma(\tau)$ will denote the Borel $\sigma$-algebra generated by $\tau$. If $A$ and $B$ are subsets of $X$ let $A+B$ represent $(A \cup B) \sim(A \cap B)$.

3. A convergence theorem. If $\theta \in \mathscr{L}_{0}$ one can define the following relation on $X: x \sim y$ if and only if $\theta_{x y}=H$. This is an equivalence relation on $X$ due to condition (b) of $\mathscr{S}$ above. Note that $\theta$ induces the structure of a continuous Menger space on $\widetilde{X}$. Let $\pi: X \rightarrow X / \sim$ be the projection map. Since $X / \sim$ is metrizable, the topology on $X$ is induced by a pseudo-metric. Consequently $\pi$ is a continuous open and closed surjection. Let $\widetilde{T}$ be the self-homeomorphism of $X / \sim$ defined by $\widetilde{T} \pi=\pi T$. 
Lemma 1. If $\theta \in \mathscr{M}_{0}$ then

$h(T)=h(\widetilde{T})$ and

$h(T)=\sup \left\{h_{\mu}(T): \mu\right.$ is a T-invariant regular probability measure on the Borel sets of $X\}$.

Proof. $\pi$ induces a Boolean isomorphism $\hat{\pi}$ between the Borel $\sigma$-algebra of $X$ and that of $X / \sim$ satisfying $\widetilde{T} \hat{\pi}=\hat{\pi} T$. Since $X / \sim$ is compact Hausdorff, we can apply the theorem of Goodwyn-DinaburgGoodman [3].

Lemma 2. Let $T$ be a bijective map of a set $X$ onto itself and let $\theta \in \mathscr{M}_{0}$. Suppose $\left\{\tau_{i}\right\}_{1}^{\infty}$ is a sequence of sub-topologies of $\tau=\tau(\theta)$. Denote $\sigma\left(\tau^{T}\right)$ by $\Sigma$ and $\sigma\left(\tau_{i}\right)$ by $\Sigma_{i}$. In addition, assume that:

$\left.{ }^{*}\right)$ Given any regular T-invariant probability measure $\mu$ on $\Sigma$, $A \in \tau$ and $\xi>0$ then there is a positive integer $N$ such that for each $i \geqq N$ there exists a set $A_{i} \in \Sigma_{i}$ satisfying $\mu\left(A+A_{i}\right)<\xi$.

Then $h\left(T, \tau_{i}^{T}\right) \rightarrow h\left(T, \tau^{T}\right)$.

Proof. We shall begin by assuming $h\left(T, \tau^{T}\right)<\infty$. Let $\varepsilon>0$ be given. Lemma 1 allows us to choose a regular $T$-invariant probability measure $\mu$ on $\Sigma$ such that $h_{\mu}(T, \Sigma) \geqq h\left(T, \tau^{T}\right)-\varepsilon / 3$. Let $P=$ $\left\{P^{1}, \cdots, P^{s}\right\} \subset \Sigma$ be a finite partition of $X$ such that $h_{\mu}(T, P) \geqq$ $h_{\mu}(T, \Sigma)-\varepsilon / 3$. Choose $\delta>0$ such that if $Q \subset \Sigma$ is a finite partition of $X$ into $s$ sets then $\left|h_{\mu}(T, P)-h_{\mu}(T, Q)\right|<\varepsilon / 3$ provided $\sum_{1}^{s} \mu\left(P^{i}+Q^{i}\right)<3 \delta$. Since $\mu$ is regular, there exist sets $A^{i} \in \tau^{T}(1 \leqq i \leqq s)$ satisfying $\mu\left(P^{i}+\right.$ $\left.A^{i}\right)<\delta / 3 s$. Choose $K>0$ and $B^{1}, \cdots, B^{s} \in \bigvee_{-K}^{K} T^{i} \tau$ such that $\mu\left(A^{i}+\right.$ $\left.B^{i}\right)<\delta / 3 s$. This can be achieved since finite intersections of $\left\{T^{i} A: A \in \tau\right.$, $i \in Z\}$ constitute a basis for $\tau^{T}$. Applying condition $\left(^{*}\right)$ of the hypothesis, there exists an integer $N>0$ such that for each $j \geqq N$ one can find sets $C^{1}, \cdots, C^{s} \in \bigvee_{i=-K}^{K} T^{i} \Sigma_{j}$ satisfying $\mu\left(B^{i}+C^{i}\right)<\delta / 3 s$ $(1 \leqq i \leqq s)$. Applying the triangle inequality one obtains $\mu\left(P^{i}+C^{i}\right)<$ $\delta / s$. Now since $\Sigma \mu\left(C^{i}\right) \leqq \Sigma\left(\mu\left(P^{i}\right)+\delta / s\right)=1+\delta$ one can construct a partition $\bar{C}=\left\{\bar{C}^{1}, \cdots, \bar{C}^{s}\right\} \subset \bigvee_{i=-K}^{K} T^{i} \sum_{j}$ of $X$ satisfying $\sum_{i=1}^{s} \mu\left(P^{i}+\bar{C}^{i}\right) \leqq$ $s(\delta / s)+2 \delta=3 \delta$. Therefore, $\left|h_{\mu}(T, \bar{C})-h_{\mu}(T, P)\right|<\varepsilon / 3$. Consequently, for all $j \geqq N, \quad h\left(T, \tau_{j}^{T}\right) \geqq h_{\mu}\left(T, \sigma\left(\tau_{j}\right)\right) \geqq h_{\mu}(T, \bar{C}) \geqq h_{\mu}(T, P)-\varepsilon / 3 \geqq$ $h_{\mu}(T, \Sigma)-2 / 3 \varepsilon \geqq h(T, \Sigma)-\varepsilon$. It remains only to note that since $\tau_{j} \subset \tau, h\left(T, \tau_{j}^{T}\right) \leqq h\left(T, \tau^{T}\right)$.

In case $h\left(T, \tau^{T}\right)=\infty$ a similar argument may be used.

Lemma 3. If $\theta \in \mathscr{M}_{0}$ and $p \in X$ then $\{U(p, \varepsilon, \lambda, \theta): \varepsilon>0, \lambda>0\}$ is a local basis for $\tau(\theta)$ at $p$.

Proof. Since $\hat{\pi}$ is an isomorphism of the Borel $\sigma$-algebra of $X$ 
with that of $\widetilde{X}$ such that $\hat{\pi}(U(p, \varepsilon, \lambda))=\widetilde{U}(\pi(p), \varepsilon, \lambda)$, it suffices to prove the lemma under the additional hypothesis that $(X, \theta)$ is a Menger space.

It was proven in Theorem 7.2 of [8] that $\{U(p, \varepsilon, \lambda): p \in X, \varepsilon>0$, $\lambda>0\}$ is a basis for $\tau(\theta)$. Let $z \in U(p, \varepsilon, \lambda)$ be given. We must show that there exist $\bar{\varepsilon}>0, \bar{\lambda}>0$ such that $U(z, \bar{\varepsilon}, \bar{\lambda}) \subset U(p, \varepsilon, \lambda)$. Suppose that for each positive integer $j$ there exists $y_{j} \in U(p, 1 / j, 1 / j) \sim$ $U(p, \varepsilon, \lambda)$. By compactness there exists a $y \in X$ and a subsequence $\left\{y_{j_{n}}\right\}$ such that $y_{j_{n}} \rightarrow y$. Now Theorem 8.1 of [8] yields $\lim \inf _{n \rightarrow \infty} \theta\left(y_{j_{n}}, z\right)=$ $\theta(y, z)$. But $\theta\left(y_{j_{n}}, z\right)(1 / m)>1-1 / m$ for $j_{n} \geqq m$. Hence $\theta(y, z)=H$ which implies $y=z$ contradicting the fact that $y \in X \sim U(p, \varepsilon, \lambda)$.

Lemma 4. If $\theta \in \mathscr{M}_{0}, \Gamma \in \mathscr{S}_{0}$, and $\theta \geqq \Gamma$ then $\tau(\theta) \subset \tau(\Gamma)$.

Proof. This follows at once from Lemma 3 together with the observation that $U(p, \varepsilon, \lambda, \theta) \supset U(p, \varepsilon, \lambda, \Gamma)$.

Lemma 5. Given $\theta \in \mathscr{L}_{0}$ and a countable dense subset $\left\{y_{i}\right\}_{1}^{\infty}$ of $(X, \tau(\theta))$ then $\left\{U\left(y_{i}, \varepsilon, \lambda\right): i \in Z^{+}, \varepsilon, \lambda \in Q^{+}\right\}$is a countable basis for $\tau(\theta)$.

Proof. This follows from an argument similar to the one given in the proof of Lemma 3.

Lemma 6. Suppose $F_{n}, F \in \mathscr{D}$ and $F_{n} \geqq F$ for all $n$. Let $F_{n}(x+)$ denote the right limit of $F_{n}$ at $x$. If $F_{n} \stackrel{D}{\rightarrow} F$ then $F_{n}(x+) \rightarrow F(x+)$ for all $x$.

Proof. Let $x \in R$ and $\varepsilon>0$. Choose $y$ to be a continuity point of $F$ such that $y>x$ and $F(y)-F(x+)<\varepsilon$. Since $F(x+) \leqq F_{n}(x+) \leqq$ $F_{n}(y)$ we have $F(x+) \leqq \lim \inf _{n \rightarrow \infty} F_{n}(x+) \leqq \lim \sup _{n \rightarrow \infty} F_{n}(x+) \leqq F(y)$. The desired conclusion now easily follows.

Theorem. Given $\left\{\theta^{i} \in \mathscr{L}_{0}: i \in Z^{+}\right\}$and $\Psi \in \mathscr{L}_{0}$ satisfying $\theta^{i} \geqq \Psi$ for each $i$ and $\theta^{i} \stackrel{D}{\rightarrow} \Psi$ then $h\left(T, \theta^{i}\right) \rightarrow h(T, \Psi)$.

Proof. Let $A \in \tau(\Psi)$ and $\varepsilon>0$ be given and let $\mu$ be a $T$-invariant probability measure on $\sigma\left(\tau(\Psi)^{T}\right)$. Since $(X, \tau(\Psi))$ is separable, Lemma 5 implies the existence of sequences $\left\{p_{i} \in A\right\},\left\{\varepsilon_{i}>0\right\}$ and $\left\{\lambda_{i}>0\right\}$ satisfying $\bigcup_{i=1}^{\infty} U\left(p_{i}, \varepsilon_{i}, \lambda_{i}, \Psi\right)=A$. Since $\mu$ is finite, there exists a positive integer $N_{1}$ such that $\mu\left(A \sim \bigcup_{i=1}^{N_{1}} U\left(p_{i}, \varepsilon_{i}, \lambda_{i}, \Psi\right)\right)<\varepsilon / 4$. Using the fact that $\bigcup_{j=1}^{\infty} U\left(p_{\imath}, \varepsilon_{i}, \lambda_{i}\left(1-2^{-j}\right), \Psi\right)=U\left(p_{i}, \varepsilon_{i}, \lambda_{i}, \Psi\right)$ we can select $0<\bar{\lambda}_{\imath}<\lambda_{\imath}$ for $1 \leqq i \leqq N_{1}$ such that $\mu\left(A \sim \bigcup_{i=1}^{N_{1}} U\left(p_{i}, \varepsilon_{2}, \bar{\lambda}_{i}, \Psi\right)\right)<$ $\varepsilon / 3$. Furthermore, since $\Psi_{p q}$ is left continuous we may choose $0<$ 
$\bar{\varepsilon}_{i}<\varepsilon_{i}$ for $1 \leqq i \leqq N_{1}$ such that $\mu\left(A \sim \bigcup_{i=1}^{N_{1}} U\left(p_{i}, \bar{\varepsilon}_{i}, \bar{\lambda}_{i}, \Psi\right)\right)<\varepsilon / 2$. Applying Lemma 6 and Egoroff's theorem, there exists a set $G \subset A$ such that $\mu(A \sim G)<\varepsilon / 2$ and $\theta_{p_{i} q}^{n}\left(\bar{\varepsilon}_{i}+\right) \rightarrow \Psi_{p_{i} q}\left(\bar{\varepsilon}_{i}+\right)$ uniformly in $q \in G$ as $n \rightarrow \infty$ for $1 \leqq i \leqq N_{1}$. As a consequence of the uniform convergence of the functions $\theta_{p_{i} q}^{n}\left(\bar{\varepsilon}_{i}+\right)$ on $G$ there exists a positive integer $N_{2}$ such that:

$$
\begin{aligned}
& U\left(p_{i}, \bar{\varepsilon}_{i}, \bar{\lambda}_{i}, \Psi\right) \cap G \subset\left\{q: \theta_{p_{i} q}^{n}\left(\bar{\varepsilon}_{i}+\right)>1-\bar{\lambda}_{i}\right\} \cap G \\
& \quad \subset\left\{q: \Psi_{p_{i} q}\left(\bar{\varepsilon}_{i}+\right)>1-\lambda_{i}\right\} \cap G \subset U\left(p_{i}, \varepsilon_{i}, \lambda_{i}, \Psi\right) \subset A
\end{aligned}
$$

for $1 \leqq i \leqq N_{1}$ and all $n \geqq N_{2}$. Then for $n \geqq N_{2}, \mu\left(A+\bigcup_{i=1}^{N_{1}} U\left(p_{i}\right.\right.$, $\left.\left.\varepsilon_{i}, \lambda_{i}, \theta^{n}\right)\right)<\varepsilon$. Lemma 4 implies that the $\left\{\tau\left(\theta^{i}\right)\right\}$ are subtopologies of $\tau(\Psi)$. An application of Lemma 2 completes the proof.

As a consequence of the theorem we obtain:

COROLlaRY. Let $\left\{d, d_{n}: n \in Z^{+}\right\}$be pseudo-metrics on the space $X$ and $T: X \rightarrow X$ be a bijection satisfying

(a) $d_{n}(x, y) \leqq d(x, y)$ for all $x, y \in X$.

(b) $d_{n}(x, y) \rightarrow d(x, y)$ as $n \rightarrow \infty$.

(c) $T$ is a self-homeomorphism of $(X, d)$ and of $\left(X, d_{n}\right)$ for each $n$.

(d) $(X, d)$ is compact.

Let $T_{n}$ denote the self-homeomorphism $T$ of the topological space $\left(X, d_{n}\right)$.

Then $h\left(T_{n}\right) \rightarrow h(T)$.

Proof. The proof follows from Theorem 5.1 of [8] where it is shown that every metric space is a continuous Menger space. Since $T_{n}$ is a homeomorphism it is easy to see that $d_{n}\left(T^{i} x, T^{i} y\right)=0$ whenever $d_{n}(x, y)=0$. Now the theorem may be applied.

We present two examples to illustrate the use of the theorem and to show that the theorem is no longer valid if one removes the condition that $\theta_{n} \geqq \theta$.

Example A. Let $Y=\left\{0,1 / n: n \in Z^{+}\right\}$be endowed with the induced topology of $I, X=Y^{Z}$ and $T: X \rightarrow X$ be the shift defined by $T\left(\left\{y_{2}\right\}\right)=\left\{y_{i+1}\right\}$. The space $X$ is metrized by:

$$
d\left(\left\{y_{i}\right\},\left\{z_{i}\right\}\right)=\sum_{-\infty}^{\infty} \frac{\left|y_{i}-z_{i}\right|}{2^{|i|}} .
$$

Subtopologies of $(X, d)$ are determined by pseudo-metrics $d_{n}$ defined by:

$$
d_{n}\left(\left\{y_{j}\right\},\left\{z_{j}\right\}\right)=\sum_{j=-\infty}^{\infty} \frac{\left|y_{j} \omega_{n}\left(y_{j}\right)-z_{j} \omega_{n}\left(z_{j}\right)\right|}{2^{|j|}}
$$


where $\omega_{n}$ is the characteristic function of $[1 / n, 1]$. Let $T_{n}$ denote the self-homeomorphism $T$ of $\left(X, d_{n}\right)$. Then $d_{n} \leqq d, d_{n} \rightarrow d, h\left(T_{n}\right)=$ $\ln n$ and $h(T)=\infty$.

Example B. Let $T$ be the shift on $X$ as above. For $n \geqq 1$ let $u_{n}$ be the pseudo-metric on $X$ given by:

$$
u_{n}\left(\left\{y_{j}\right\},\left\{z_{j}\right\}\right)=\sum_{j=-\infty}^{\infty} \frac{\left|t_{n}\left(y_{j}\right)-t_{n}\left(z_{j}\right)\right|}{2^{|j|}}
$$

where

$$
t_{n}(s)=\left\{\begin{array}{l}
\frac{1}{n} \text { if } s \geqq \frac{1}{n} \\
s \text { otherwise } .
\end{array}\right.
$$

Then $u_{n} \rightarrow u$ as $n \rightarrow \infty$, where $u(x, y)=0$ for all $x, y \in X$. Now $h\left(T, u_{n}\right)=\infty$ but $h(T, u)=0$.

We conclude with the following speculation:

Conjecture. Given $\left\{\theta^{i} \in \mathscr{C}_{0}: i \in Z^{+}\right\}$and $\Psi \in \mathscr{C}_{0}$ satisfying $\theta^{i} \stackrel{D}{\rightarrow} \Psi$ then

$$
\liminf _{i \rightarrow \infty} h\left(T, \theta^{i}\right) \geqq h(T, \Psi)
$$

\section{REFERENCES}

1. R. L. Adler, A. G. Konheim, and M. H. McAndrew, Topological entropy, Trans. Amer. Math. Soc., 114 (1965), 309-319.

2. P. Billingsley, Ergodic Theory and Information, Wiley and Sons, New York, 1965.

3. T. N. T. Goodman, Relating topological entropy and measure entropy, Bull. London Math. Soc., 3 (1971), 176-180.

4. M. Loève, Probability Theory, $3^{\text {rd }}$ ed., Van Nostrand Co., Princeton, N.J., 1963.

5. K. Menger, Statistical metrics, Proc. Nat. Acad. Sci., 28 (1942), 535-537.

6. W. Parry, Entropy and Generators in Ergodic Theory, Benjamin, New York, 1969.

7. B. Schweizer, Probabilistic metric spaces-the first 25 years, The N. Y. Statistician, 19 (1967), 3-6.

8. B. Schweizer and A. Sklar, Statistical metric spaces, Pacific J. Math., 10 (1960), 313-334.

9. B. Schweizer, A. Sklar, and E. Thorp, The metrization of statistical metric spaces, Pacific J. Math., 10 (1960), 673-675.

Received November 27, 1972.

UNIVERSITY OF VIRGINIA 


\section{PACIFIC JOURNAL OF MATHEMATICS}

\section{EDITORS}

RICHARD ARENS (Managing Editor)

University of California

Los Angeles, California 90024
J. DUGUNDJI*

Department of Mathematics

University of Southern California

Los Angeles, California 90007

D. Gilbarg and J. Milgram

Stanford University

Stanford, California 94305
University of Washington

Seattle, Washington 98105

ASSOCIATE EDITORS
E. F. BECKENBACH
B. H. NeumanN
F. WoLF
K. YosHIDA

\section{SUPPORTING INSTITUTIONS}

\author{
UNIVERSITY OF BRITISH COLUMBIA \\ CALIFORNIA INSTITUTE OF TECHNOLOGY \\ UNIVERSITY OF CALIFORNIA \\ MONTANA STATE UNIVERSITY \\ UNIVERSITY OF NEVADA \\ NEW MEXICO STATE UNIVERSITY \\ OREGON STATE UNIVERSITY \\ UNIVERSITY OF OREGON \\ OSAKA UNIVERSITY
}

\author{
UNIVERSITY OF SOUTHERN CALIFORNIA \\ STANFORD UNIVERSITY \\ UNIVERSITY OF TOKYO \\ UNIVERSITY OF UTAH \\ WASHINGTON STATE UNIVERSITY \\ UNIVERSITY OF WASHINGTON
* * * *
AMERICAN MATHEMATICAL SOCIETY \\ NAVAL WEAPONS CENTER
}

The Supporting Institutions listed above contribute to the cost of publication of this Journal, but they are not owners or publishers and have no responsibility for its content or policies.

Mathematical papers intended for publication in the Pacific Journal of Mathematics should be in typed form or offset-reproduced, (not dittoed), double spaced with large margins. Underline Greek letters in red, German in green, and script in blue. The first paragraph or two must be capable of being used separately as a synopsis of the entire paper. Items of the bibliography should not be cited there unless absolutely necessary, in which case they must be identified by author and Journal, rather than by item number. Manuscripts, in duplicate if possible, may be sent to any one of the four editors. Please classify according to the scheme of Math. Rev. Index to Vol. 39. All other communications to the editors should be addressed to the managing editor, or Elaine Barth, University of California, Los Angeles, California, 90024.

100 reprints are provided free for each article, only if page charges have been substantially paid. Additional copies may be obtained at cost in multiples of 50 .

The Pacific of Journal Mathematics is issued monthly as of January 1966. Regular subscription rate: $\$ 72.00$ a year (6 Vols., 12 issues). Special rate: $\$ 36.00$ a year to individual members of supporting institutions.

Subscriptions, orders for back numbers, and changes of address should be sent to Pacific Journal of Mathematics, 103 Highland Boulevard, Berkeley, California, 94708.

PUBLISHED BY PACIFIC JOURNAL OF MATHEMATICS, A NON-PROFIT CORPORATION

Printed at Kokusai Bunken Insatsusha (International Academic Printing Co., Ltd.), 270, 3-chome Totsuka-cho, Shinjuku-ku, Tokyo 160, Japan.

* C. R. DePrima California Institute of Technology, Pasadena, CA 91109, will replace J. Dugundji until August 1974.

Copyright (C) 1973 by Pacific Journal of Mathematics

Manufactured and first issued in Japan 


\section{Pacific Journal of Mathematics}

\section{Vol. 51, No. $2 \quad$ December, 1974}

Robert F. V. Anderson, Laplace transform methods in multivariate spectral theory .................................................. 339

William George Bade, Two properties of the Sorgenfrey plane . . . . . . . . . . . . 349

John Robert Baxter and Rafael Van Severen Chacon, Functionals on continuous

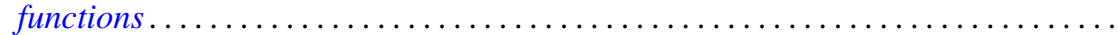

Phillip Wayne Bean, Helly and Radon-type theorems in interval convexity

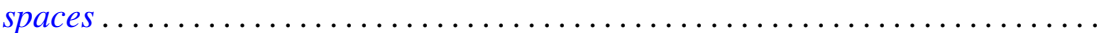

James Robert Boone, On k-quotient mappings $\ldots \ldots \ldots \ldots \ldots \ldots \ldots \ldots \ldots$

Ronald P. Brown, Extended prime spots and quadratic forms . . . . . . . . . . . .

William Hugh Cornish, Crawley's completion of a conditionally upper continuous lattice .............................................

Robert S. Cunningham, On finite left localizations ...................

Robert Jay Daverman, Approximating polyhedra in codimension one spheres

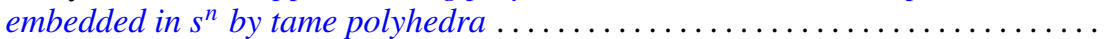

Burton I. Fein, Minimal splitting fields for group representations . . . . . . . . . . . .

Peter Fletcher and Robert Allen McCoy, Conditions under which a connected

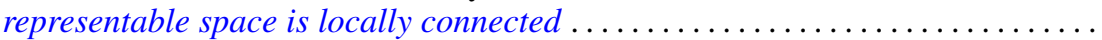

Jonathan Samuel Golan, Topologies on the torsion-theoretic spectrum of a noncommutative ring...

Manfred Gordon and Edward Martin Wilkinson, Determinants of Petrie matrices.

Alfred Peter Hallstrom, A counterexample to a conjecture on an integral condition for determining peak points (counterexample concerning peak points)........

E. R. Heal and Michael Windham, Finitely generated $F$-algebras with applications to Stein manifolds.

Denton Elwood Hewgill, On the eigenvalues of a second order elliptic operator in an unbounded domain ............................

Charles Royal Johnson, The Hadamard product of $A$ and $A^{*}$.

Darrell Conley Kent and Gary Douglas Richardson, Regular completions of Cauchy spaces.

Alan Greenwell Law and Ann L. McKerracher, Sharpened polynomial approximation

Bruce Stephen Lund, Subalgebras of finite codimension in the algebra of analytic functions on a Riemann surface. .

Robert Wilmer Miller, TTF classes and quasi-generators . .

Roberta Mura and Akbar H. Rhemtulla, Solvable groups in which every maximal partial order is isolated ....

Isaac Namioka, Separate continuity and joint continuity...

Alan Saleski, Entropy of self-homeomorphisms of statistical pseudo-metric

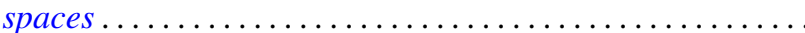

H. A. Seid, Cyclic multiplication operators on $L_{p}$-spaces .....

H. B. Skerry, On matrix maps of entire sequences ............

John Brendan Sullivan, A proof of the finite generation of invariants of a normal

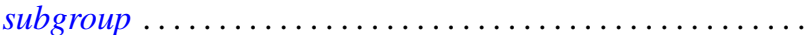

John Griggs Thompson, Nonsolvable finite groups all of whose local subgroups are

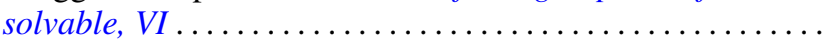

